\title{
Measurement of Poloidal Flow Profiles Using a Mach Probe Array in HYBTOK-II Tokamak with RMP Fields
}

\author{
Masaaki OKAMOTO, Satoru SAKAKIBARA ${ }^{1)}$, Naoya YOSHIMOTO²), Takuya OHNO²) \\ and Noriyasu $\mathrm{OHNO}^{2}$ ) \\ National Institute of Technology, Ishikawa College, Tsubata, Ishikawa 929-0392, Japan \\ ${ }^{1)}$ National Institute for Fusion Science, Toki, Gifu 509-5292, Japan \\ ${ }^{2)}$ Graduate School of Engineering, Nagoya University, Nagoya 464-8603, Japan
}

(Received 30 March 2017 / Accepted 2 May 2017)

\begin{abstract}
In magnetic confinement plasmas, plasma rotation plays an important role in the penetration and propagation processes of external RMP fields. To investigate the relation between the penetration of RMP fields and plasma flow, we have created a new type of Mach probe array to measure plasma flow profiles. The experimental results show that plasma flow around the $q=3$ resonant surface decreases with increases in RMP fields.
\end{abstract}

(C) 2017 The Japan Society of Plasma Science and Nuclear Fusion Research

Keywords: Mach probe array, plasma flow, RMP fields, HYBTOK-II tokamak

DOI: $10.1585 /$ pfr.12.1202027

In magnetic confinement plasmas, resonant magnetic perturbation (RMP) fields are expected to control the transport and MHD instability in the edge region. In several tokamaks, the investigations on the effect of RMP fields in the plasma have indicated that the penetration of RMP fields into the plasma depends on the shielding current at the resonant surface [1-3]. Therefore, the plasma rotation in the vicinity of the resonant surface plays a crucial role in the penetration process of RMP fields. In this study, the plasma flow profile of HYBTOK-II tokamak plasma in the presence of RMP fields was measured via a new Mach probe array to investigate the relation between the penetration of RMP fields and plasma flow.

The major and limiter radii of HYBTOK-II tokamak are $40 \mathrm{~cm}$ and $11 \mathrm{~cm}$, respectively. The RMP coils are installed at $R=68 \mathrm{~cm}$ have $n=1$ mode structure, as shown in Fig. 1 (a). The RMP fields are rotated in the toroidal direction by changing the phase difference of the two coil currents. The phase difference of coil currents was set to $90^{\circ}$ and the rotational direction was clockwise. A new Mach probe array was installed in the side port of \#7, as shown in Fig. 1 (b). This probe, shown in Fig. 1 (c), has high spatial resolution, which is $2 \mathrm{~mm}$ and the channel number is 30 , because it has the laminar structure of ceramics with signal lines. In this study, ion saturation currents at the upper and lower surfaces of the probe were measured to estimate the poloidal flow.

Figure 2 shows the waveforms of RMP coil currents, ion saturation currents measured on the upper and lower surfaces of Mach probe, and the poloidal magnetic field fluctuation of $m=3$ mode on the low field side. The RMP fields were applied between $t=9-17 \mathrm{~ms}$ and the

author'se-mail: okamoto@ishikawa-nct.ac.jp coil current $I_{\mathrm{RMP}}$ and frequency were $100 \mathrm{~A}$ and $3 \mathrm{kHz}$, respectively. In Fig. 2 (b), the waveform of the ion saturation current shows a moving average of $0.1 \mathrm{~ms}$. Because the ion saturation current at the upper surface was larger than that at the lower surface, the poloidal flow was in the ion diamagnetic direction. In Fig. 2 (c), the waveform of $m=3$ mode, after subtracting the signal without the plasma, was calculated using 8 magnetic coil signals and the following

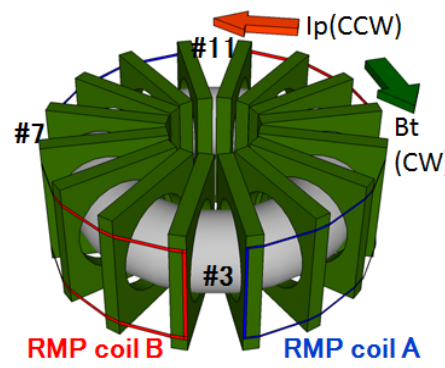

(a)

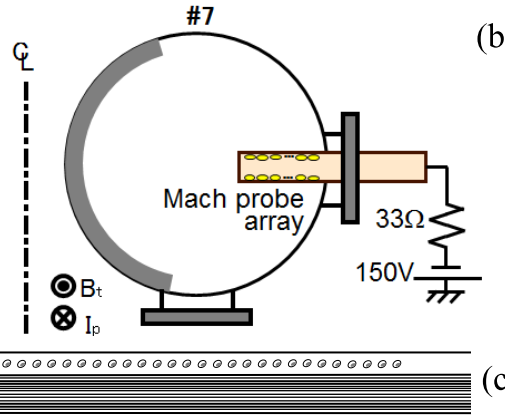

(b)

(c)

Fig. 1 Structure of the RMP coils installed in HYBTOK-II (a), schematic of Mach probe array measurement system (b), and schematic of Mach probe array (c). 


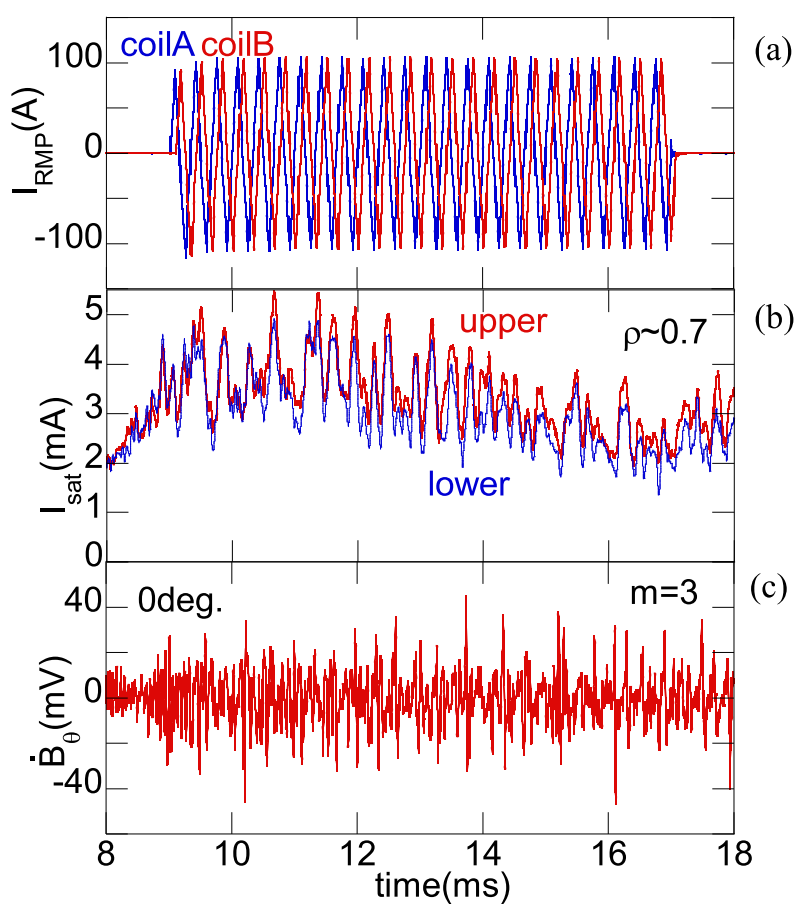

Fig. 2 Time evolution of RMP coil currents (a), ion saturation currents at upper and lower surfaces of Mach probe (b) and $m=3$ mode signal estimated from poloidal magnetic fluctuations (c).

Fourier expansion equations.

$$
\begin{aligned}
& \dot{B}_{\theta}(\theta)=\sum\left(a_{m} \cos m \theta+b_{m} \sin m \theta\right), \\
& a_{m}=\frac{1}{\pi} \int \dot{B}_{\theta}(\theta) \cos m \theta d \theta, \\
& b_{m}=\frac{1}{\pi} \int \dot{B}_{\theta}(\theta) \sin m \theta d \theta .
\end{aligned}
$$

Here, $\dot{B}_{\theta}, \theta$, and $m$ are the time derivative of the poloidal magnetic field, the poloidal angle, and the poloidal mode number, respectively. The signal in Fig. 2 (c) was estimated by substituting $\theta=0^{\circ}$ and $m=3$ in Eq. (1).

Figure 3 shows the power spectra of the ion saturation current and $m=3$ mode signal, calculated by applying a fast Fourier transform for $t=10-14 \mathrm{~ms}$ to the data in Fig. 2. When RMP coil current was $20 \mathrm{~A}$, the main frequency components of the magnetic signal and ion saturation current fluctuation appeared in the vicinity of $f=10 \mathrm{kHz}$, and seemed to be MHD fluctuations. These fluctuations were also found at $I_{\mathrm{RMP}}=50$ and $100 \mathrm{~A}$. On the contrary, the $3 \mathrm{kHz}$ component, which was the driving frequency of RMP fields, was not found at $I_{\mathrm{RMP}}=20 \mathrm{~A}$; Therefore, such RMP fields have a negligible influence on the plasma. Because the $3 \mathrm{kHz}$ components of $m=3$ mode and ion saturation current increased with the increasing of RMP coil current, we consider that the magnetic island formed and grew around the $q=3$ resonant surface.

Figure 4 shows the time-averaged profiles of Mach number and safety factor $q$ for $t=10$ - 14 ms. In Fig. 4 (a),

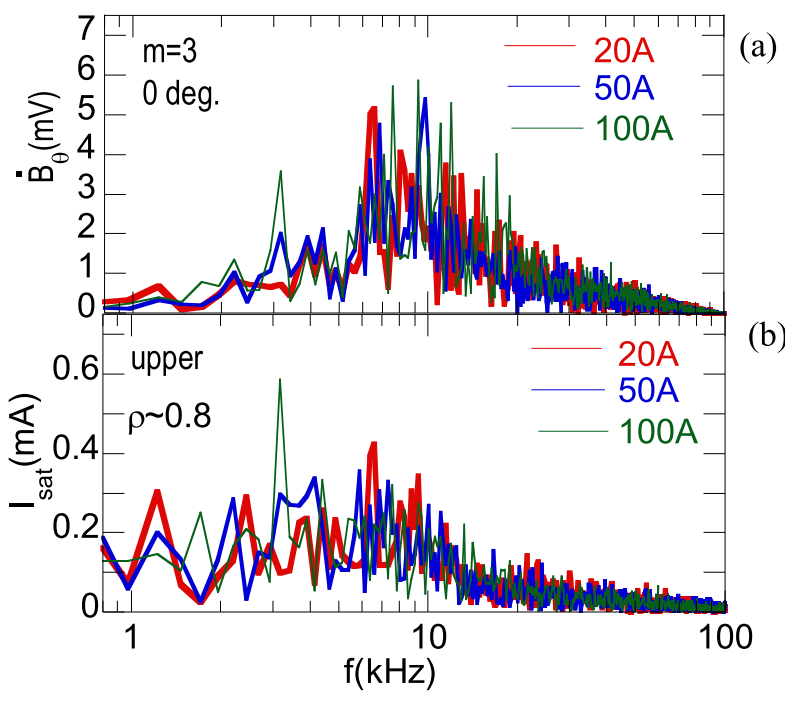

Fig. 3 FFT power spectra of $m=3$ mode signals (a) and ion saturation currents measured at the upper surface of Mach probe (b).

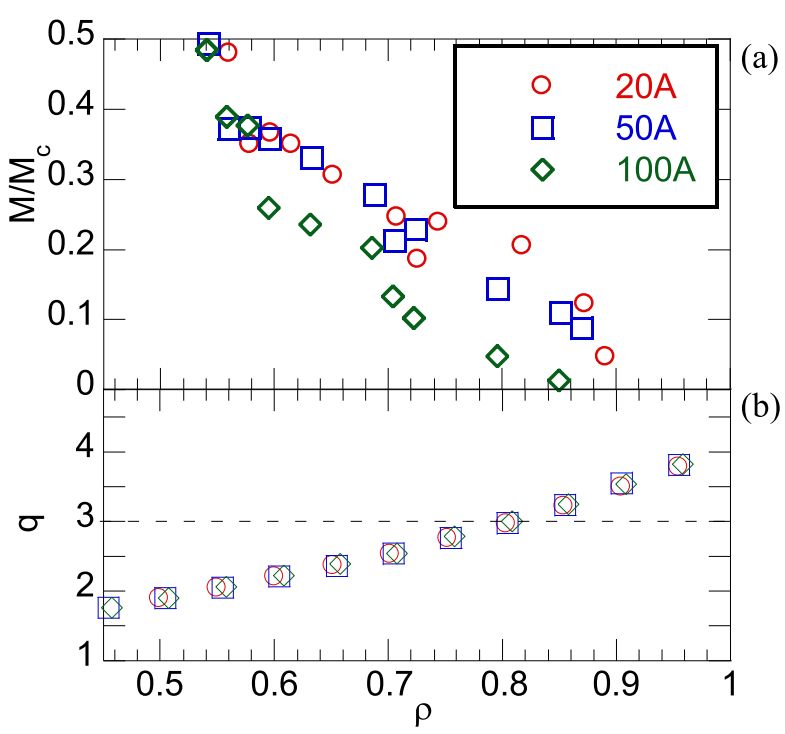

Fig. 4 Profiles of poloidal Mach number estimated from ratio of ion saturation currents at up and downstream (a) and safety factor (b).

the Mach number depends on the poloidal flow and positive values indicate the ion diamagnetic direction. The Mach number $M$ was calculated as follows:

$$
M / M_{\mathrm{c}}=\ln \left(j_{\mathrm{u}} / j_{\mathrm{d}}\right)
$$

Here, the coefficient $M_{\mathrm{c}}=c_{\mathrm{s}} / 4 v_{\mathrm{t}}, j_{\mathrm{u}}$ and $j_{\mathrm{d}}$ are the upstream and downstream current densities, respectively [4]. The value of $M / M_{\mathrm{c}}$ at $I_{\mathrm{RMP}}=20 \mathrm{~A}$ monotonically increased toward the center of the plasma. The value of $M / M_{\mathrm{c}}$ around the $q=3$ surface at $I_{\mathrm{RMP}}=50 \mathrm{~A}$ was smaller than at $I_{\mathrm{RMP}}=20 \mathrm{~A}$, and the value at $I_{\mathrm{RMP}}=100 \mathrm{~A}$ was even smaller. In conclusion, we found that the poloidal 
flow around the $q=3$ surface decreased when RMP fields were applied. In addition, we speculate that $\mathrm{E} \times \mathrm{B}$ and diamagnetic drift velocities decrease with the flattening of the electron temperature and density profiles in the $m / n=3 / 1$ magnetic island.
[1] T.C. Hender et al., Nucl. Fusion 32, 2091 (1992).

[2] R.J. La Haye et al., Phys. Fluids B 4, 2098 (1992).

[3] S.Takamura et al., Nucl. Fusion 43, 393 (2003).

[4] I.H. Hutchinson, Phys. Plasmas 9, 1832 (2002). 